\title{
Countermeasure Research of the Causes of the Lack of Rural Aged-Education in Northern Jiangsu
}

\author{
Dongsheng Yi, and Guolian Wang \\ Huaiyin Institute of Technology, Huai'an, Jiangsu \\ Huaiyin Institute of Technology, Huai'an, Jiangsu \\ yidongsheng91@163.com
}

\begin{abstract}
Keywords: Rural Areas in Northern Jiangsu, Aged-Education, Cause Analysis, Countermeasure Study

Abstract. The aging of population has become a common and urgent issue that needs to be solved by the human beings. The world has reached a consensus that we should help the elderly people adapt to the social transformation and integrate into the society, strengthen their self-esteem, and develop their potentials via education. After grasping the present situation and existing problems of rural elderly education, this subject group takes Northern Jiangsu as an example, a less developed area in Jiangsu Province, from an education perspective, attempt to implement cause analysis to explore the ways that the aged could participate in the education and to solve and prevent the social problems caused by aging populations especially the accelerated increase in the aged population in rural areas and provide theoretical basis and practical experience for the aged-education in rural China.
\end{abstract}

\section{Introduction}

The aging of population has become a common problem across the world. Jiangsu Province, a province in the eastern China with a relatively fast development in the modernization process, is also facing the challenge of aging population.

According to the report on The Information and Development of The Elderly People in Jiangsu Province from the Perspective of Population Composition, "Jiangsu has a larger elderly population as well as a faster aging process compared to the other areas"; while "The percentage of elderly population of rural areas is far greater than that of urban areas", and at the same time we have "An increasing number of Empty-Nester aged people".

Faced with this severe reality, in the urban areas in Jiangsu as well as the rural areas in Southern Jiangsu, the living security system for elderly people has improved a lot, and the stereotype image of aged people has been changing gradually. With a growing awareness of keeping accepting education along the way, the aged population are also rebuilding their sense of self-identity and therefore their happiness index is increasing at the same time.

However in the rural area of Northern Jiangsu, the development of education for aged people is relatively slow, and some villages and towns are still blinding spots according to some related quantitative analysis and field survey. The only way to solve this problem is to analyze the possible causes. After research the subject group believe the main reasons which give rise to this issue are:

Confounded by Traditional Thoughts: Through the long process of social development, because a lot of the aged people are confounded by the traditional thoughts that "At fifty I knew the mandate of heaven; at sixty my ear was obedient;" and they usually have a poor self-cognition, the elderly people are more easily to be affected by this stereotype image. The subject group conducted a random survey and found that most people in rural areas of Northern Jiangsu care little about the further education for the aged people, even the percentage for people who would support the aged people in their family is only $5.2 \%$. Among all the elderly people taking this survey, when they were asked the question that "If you have the opportunity to participate in senior education or training what do you want to participate in", about $67.3 \%$ of them answered "It's embarrassing to participate in training in their age".

Relatively Backwards in Economic Development: Northern Jiangsu rural areas are relatively backwards in economic development, and the fund for the aged-education in these areas are much less 
than other place in Jiangsu. The subject group found that about $21.9 \%$ of the interviewers are not able to join in any education activities because of shortage of money.

Lack of Attention from Local Governments: Local governments do not pay enough attention to the aged-education in rural areas of Northern Jiangsu, details are as follows: first of all, not many education sessions are held or available for the elderly people to take. The researchers find that about only $19.8 \%$ of the villages and towns have ever held education activities targeted at the elderly before; among all the aged people interviewed about $35.9 \%$ of them have never got the chance to take part in any education or training sessions; about $39.8 \%$ of the population would join in those activities once in a while. Secondly, public facilities for the aged-education have been in a severe shortage. The percentage for villages with education institutions specialize in the aged people in Northern Jiangsu takes up to around 19\%, less than one fifth. What's more, the development of teaching staff is very slow. The existing education and training activities for the aged people in rural areas of Northern Jiangsu fail to satisfy the needs for elderly people of richening their spiritual and cultural life.

\section{Solution}

Free from Prejudices, Overcome Cognitive Disorder on the Senior Citizens Participating in Further Education: In traditional culture, "old" has become a pronoun for weak, useless and lack of creativity. People in the old times also believed that the aged people could not live unless they rely on the society or the young people.

Nowadays this logic has also been greatly affected by the modern Theory of Evolution, which has deepened the prejudices on the elderly people. It is true that become old would make people's body weaker, but it's not a sign for being useless. The above prejudice not only is largely caused by the society but also confound the concept for old and the aged. Because of the fact that the health condition of the aged people is not as good as before will not affect their mind, the famous ancient Roman philosopher Cicero spoke sternly and forcefully to refute the opinion that "being old is being useless and lack of creativity" in his article On Old Age, On Friendship and On Divination. He believes that after the young age when people being young and don't understand much about how to behave properly, their life goal would gradually become clearer during middle age, and people would have more time to learn knowledge in old age. At the same time, their mind and thoughts would be more mature after all the reflection, accumulation and summarization in the early ages.

Think of Ways to Tamp the Economic Foundation for The Aged to Accept Education: Since the Eighteenth Meeting, China has improved the social security level for the elderly people. However there's still huge difference in the social security level between Northern Jiangsu and the southern and middle part of Jiangsu province because of the development of local economy. Social benefits for the aged people is comparatively less in rural area in Northern Jiangsu and most of them would have to rely on their children to solve this problem, which makes the elderly people the last one of the economic chain.

Via statistics on the monthly expenses data of the aged people, the subject group has discovered that the percentage for the aged population in rural areas spend less than $100 \mathrm{RMB}$ in total every month takes up to $21 \%$, the percent for the aged people who spend more than 100 RMB and less than 200 RMB takes up to $29.7 \%$, spend more than 200 and less than 300 RMB is about $16.5 \%$ and spend more than 300 RMB is only $32.8 \%$ in Northern Jiangsu. This fact has stopped them from accepting further education to some extent.

Majority of the aged people spend less than $300 \mathrm{RMB}$ on education every month, and the percentage of the population don't spend any money on education is over $21.9 \%$. When interviewed with the question that "What is the main obstacle for you to react with senior education?" about $14.1 \%$ of the elderly people answered with the reason "High expense". The subject group believe that we could do the below things to overcome this obstacle:

Firstly, we need to call for the overall planning by governments, advise them to take into account of the education expense of the aged population in the local financial budget; secondly, try to increase the 
income of the aged people; besides, we would need to implement appropriate interval control and balance the education funding among areas; moreover, we would need help from society support and raise funds from various sources.

Let Government Take the Lead, Build Appropriate and Sufficient System: The national machinery has always been on the top of superstructure, played a very important role in the culture. At present governments at all levels do not pay enough attention to the aged-education and this phenome is especially prominent in the rural areas of Northern Jiangsu. To solve this problem governments at all levels need to make great efforts to defuse the social crisis which might be caused by the aging of population, which is also their duty. We could proceed from the following aspects:

First of all, we need to update our concepts, at the height of the strategy to put the aged-education and nine-year compulsory education at the same position. Then we need to encourage the local governments to take on the responsibility to develop the education targeted at the aged people, incorporate the assessment result into the system, improve the supervising mechanism and facilities, and make policies to encourage the elderly people to participate in the education and training activities. Besides, local governments should increase financial investments, mobilizes all sides, reform financing system, and attract social capitals to take part in the construction of the aged-education in villages and towns.

Last but not least, we need to enhance the construction of teaching staff for the aged-education in rural areas in Northern Jiangsu. Teaching staff is the fundamental guarantee for development of education. Currently in Northern Jiangsu rural area there's no teacher who specialize in senior education and even it would not be easy to find a part-time teacher. This is far from meeting the educational needs of the elderly people in Northern Jiangsu rural areas. To resolve this contradiction we need to study from the successful cases which start up the education for farmers in the early years, encourage great people who aspire to farmer education like Shuming Liang, Yangchu Yan and so on to dedicate into the aged education, offer preferential treatment, and improve the training and selection system for the aged education team.

While completing the above work, based on the realities of situation in rural Northern Jiangsu, we could proceed in two steps, the first step would be to encourage highly educated social workers and part-time teachers to help with education for the elderly population; the second one would be to combine local training and importing, gradually reinforce the teaching team for the aged-education in rural Northern Jiangsu.

Develop Diverse Educational Activities According to Actual Conditions: The underlying purpose for education is to tailor the knowledge and skills for the subject educated. Only in this way can we fulfill the goal that teaching benefits teachers as well as students.

The specialty of the subject of the aged-education in rural areas has determined that the types of education need to be flexible and versatile. The subject group has found that the aged people interviewed have different health conditions, living environment and experience, as well as huge differences in their knowledge structures, hobbies and interests, and cognitive ability.

As a result, while learning from the rural senior education in those developed areas we should also set up different education methods and contents according to the different characteristics of the aged people in rural Northern Jiangsu. Detailed methods could be: firstly, we could provide various activities according to the locations; secondly we need to realize the diversification of education content and teach students according to their aptitude. We need to apply the " $T$ " teaching structure when teaching the aged people in rural areas, broaden the scope in the content, and specialize the content based on the their characteristics. To solve this problem, we would need to get to know more about the aged people in rural areas and listen to their educational needs.

\section{Conclusions}

As a conclusion of the above, the aged-education in rural areas especially in those economic less-developed region still has a long way to go, which needs joint efforts of government, society and 
the aged population. While implement "outer education" into villages and towns, we should also promote the development of "inner education". The elderly people should have something to use, something to accomplish and something to enjoy on the basis on "being old and well learned", which will not only provide more labor forces to support society sustainable development and boost the economic and cultural development in Jiangsu province, but will also improve living quality for the elderly people, prevent mental health problems when growing old, and satisfy their hope to realize self-fulfillment. Only in this way can we give full play to the advanced cultural resources of rural areas and make up the defect of the short board at the same time, speed up the new countryside construction in a better way and provide referential theoretical evidence and practical experience for realizing rural-urban integration.

\section{References}

[1] (Ancient Rome) Marcus Tullius Cicero, On Old Age, On Friendship and On Divination, edited by Yicun Xu, Beijing, China, China Commence and Trade Press,(1998). 